\title{
Strain manipulation of Majorana fermions in graphene armchair nanoribbons
}

\author{
Zhen-Hua Wang, ${ }^{1,2}$ Eduardo V. Castro, ${ }^{1,3}$ and Hai-Qing Lin ${ }^{1}$ \\ ${ }^{1}$ Beijing Computational Science Research Center, Beijing 100084, China \\ ${ }^{2}$ Institute for Quantum Science and Engineering and Department of Physics, \\ Southern University of Science and Technology, Shenzhen 518055, China and \\ ${ }^{3}$ CeFEMA, Instituto Superior Técnico, Universidade de Lisboa, Av. Rovisco Pais, 1049-001 Lisboa, Portugal
}

\begin{abstract}
Graphene nanoribbons with armchair edges are studied for externally enhanced, but realistic parameter values: enhanced Rashba spin-orbit coupling due to proximity to a transition metal dichalcogenide like $\mathrm{WS}_{2}$, and enhanced Zeeman field due to exchange coupling with a magnetic insulator like EuS under applied magnetic field. The presence of s-wave superconductivity, induced either by proximity or by decoration with alkali metal atoms like $\mathrm{Ca}$ or $\mathrm{Li}$, leads to a topological superconducting phase with Majorana end modes. The topological phase is highly sensitive to the application of uniaxial strain, with a transition to the trivial state above a critical strain well below $0.1 \%$. This sensitivity allows for real space manipulation of Majorana fermions by applying nonuniform strain profiles. Similar manipulation is also possible by applying inhomogeneous Zeeman field or chemical potential.
\end{abstract}

PACS numbers: 62.20.D-,73.20.-r,73.22.Pr,74.45.+c,74.78.Na

Introduction. - Majorana fermions - particles which are their own anti-particles [1] - have recently been the subject of intense research due to the real prospect of realizing such exotic particles in condensed matter platforms [2, 3]. Signatures of Majorana fermions have already been found in semiconducting nanowires with strong spin-orbit coupling (SOC) in proximity to a superconductor [4 11], at the end of atomic iron chains on the surface of a superconductor [12, 13], and recently in the hybrid system of a quantum anomalous Hall insulator coupled with a superconductor as one-dimensional chiral modes [14]. These condensed matter Majorana fermions occur as zero energy quasiparticles when topological superconductivity sets in the system. These quasiparticles, so-called Majorana zero modes, obey non-Abelian statistics [15, 16] and are seen as promising building blocks to realize decoherence free topological quantum computation [17, 18]. The underlaying condensed matter support of the bounded Majorana zero modes will certainly play a key role, dictating how easily Majorana fermions can be braided, or manipulated in general. Graphene, with its highly tunable properties, is a tempting platform.

Apart from graphene's unconventional behavior [19] and potential for applications in many different areas (energy [20], friction [21], biology [22], to mention a few), it has not been recognized as an adequate host for Majorana zero modes. A key ingredient in this context is the ability to induce topological superconductivity in the host system [23 27]. This can be achieved by proximity to a normal superconductor and applied magnetic field if the system has a sizable SOC, as is the case of the semiconducting nanowires used in recent experiments [4 11]. Graphene has a vanishing SOC [28, 29], so topological superconductivity seems to be ruled out based on the standard ingredients.

This state of affairs have changed completely in recent

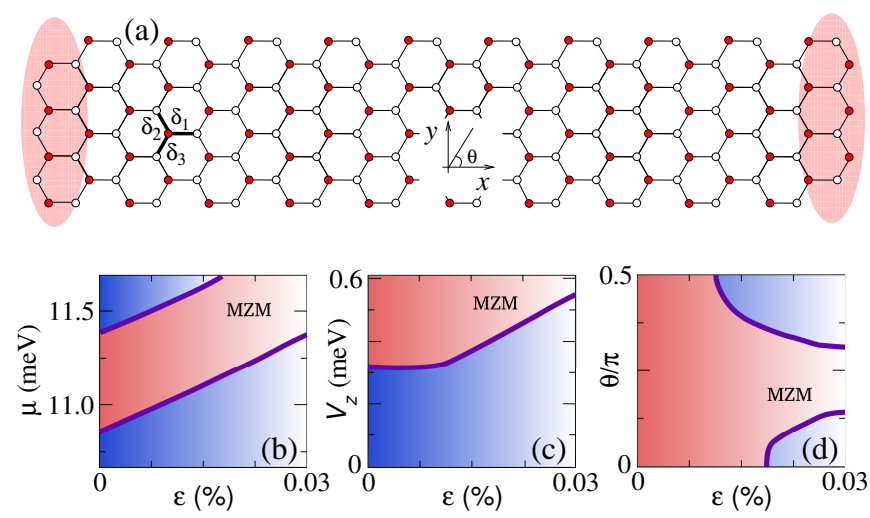

Figure 1: (a) Sketch of a graphene armchair ribbon with Majorana zero modes at both ends realized in the topological region of the phase diagram (b)-(d). Phase diagram in the plane of chemical potential $\mu$ (b), Zeeman field $V_{z}$ (c), and strain direction $\theta(\mathrm{d})$, vs strain $\varepsilon$. The topological phase with Majorana zero modes corresponds to the MZM region in the figures.

years, when an enhancement of SOC by several orders was shown by two different methods: proximity to high SOC transition metal dichalcogenides like $\mathrm{WS}_{2}$ [30 33], and via graphene hydrogenation [34, 35]. Moreover, the other basic ingredient to achieve topological superconductivity in the standard scheme, namely a magnetic field induced Zeeman field, was recently shown to achieve values comparable to those in Rashba nanowires like InAs, despite the much smaller $g$-factor in graphene. This has been achieved through a strong magnetic exchange coupling which occurs between graphene and the magnetic insulator EuS [36], clearly showing that graphene's tunability applies to a great variety of parameters.

In the present paper, we show that topological superconductivity can be realized in armchair graphene 
nanoribbons [Fig. 1(a)] with externally enhanced, but realistic parameter values. As a two dimensional membrane embedded in three-dimensional space, graphene can easily develop built-in strain which we also consider here. Indeed, built in strain of order $0.01 \%-0.1 \%$ has been reported in suspended samples [37, 38]. For supported samples, depending on the substrate, this value can be even larger, as is the case of $\mathrm{SiO}_{2}$ [39]. Taking into account the effect of strain we obtained the phase diagrams shown in Fig. 1(b)-1(d) in the plane of uniaxial strain versus chemical potential $\mu$, Zeeman field $V_{z}$, and strain direction $\theta$. The topological phase is robust for realistic values of the parameters, and is also very sensitive to strain, which can be used to tune the transition. This leads to the possibility of real space manipulation of Majorana zero modes by applying non-uniform strain, as is shown in the present work.

Model and methods. -We model $p_{z}$ electrons in graphene with a tight binding Hamiltonian,

$$
\begin{aligned}
H= & -t \sum_{\mathbf{r}, \boldsymbol{\delta}, \sigma} c_{\mathbf{r}, \sigma}^{\dagger} c_{\mathbf{r}+\boldsymbol{\delta}, \sigma}+i \lambda \sum_{\mathbf{r}, \boldsymbol{\delta}} \sum_{\sigma, \sigma^{\prime}} c_{\mathbf{r}, \sigma}^{\dagger}(\hat{\boldsymbol{\delta}} \times \boldsymbol{\sigma})_{z}^{\sigma \sigma^{\prime}} c_{\mathbf{r}+\boldsymbol{\delta}, \sigma^{\prime}} \\
& +V_{Z} \sum_{\mathbf{r}, \sigma, \sigma^{\prime}} c_{\mathbf{r}, \sigma}^{\dagger} \sigma_{\alpha}^{\sigma \sigma^{\prime}} c_{\mathbf{r}, \sigma^{\prime}}+\Delta \sum_{\mathbf{r}}\left(c_{\mathbf{r}, \uparrow}^{\dagger} c_{\mathbf{r}, \downarrow}^{\dagger}+h . c .\right),(1)
\end{aligned}
$$

where $c_{\mathbf{r}, \sigma}^{\dagger}$ creates an electron with spin $\sigma$ at site $\mathbf{r}$ of the honeycomb lattice, and the three vectors $\boldsymbol{\delta}$ connect nearest neighbor atoms as shown in Fig. 1(a). The first term in Eq. (11) is graphene's minimum tight binding Hamiltonian with $t \approx 3 \mathrm{eV}$ for the nearest neighbor hopping integral [19]. The second term is the Rashba SOC [40], where we defined the unit vector $\hat{\boldsymbol{\delta}}=\boldsymbol{\delta} / a$ with $a$ for the carbon-carbon distance. The third term is the Zeeman coupling induced by an in-plane magnetic field $\mathbf{B}=B \vec{e}_{\alpha}$ in the direction $\alpha=x, y$, and the last term is the induced $s$-wave superconductivity, as in Ref. [41].

Strain is introduced through changes on the matrix elements of the Hamiltonian connecting nearest neighbor sites. For the hopping parameter the change reads 42 , 43],

$$
t_{\hat{\boldsymbol{\delta}}} \rightarrow t_{\hat{\boldsymbol{\delta}}}(1-\beta(\hat{\boldsymbol{\delta}} \cdot \nabla) \mathbf{u} . \hat{\boldsymbol{\delta}},
$$

where $\mathbf{u}$ is the deformation field, and $\beta=-\partial \ln t / \partial \ln a \approx$ 2. Here we consider uniaxial strain applied along a direction specified by $\theta$ [see Fig. 1(a)], and parametrize the strain tensor $u_{i j}=\left(\partial_{j} u_{i}+\partial_{i} u_{j}\right) / 2$ appearing in Eq. (2) in terms of a single parameter $\varepsilon$ [44]. A similar strain induced change can be used for Rashba SOC [45], but no quantitative changes can be noticed in the phase diagram.

The topological and trivial phases are identified by: the presence of a finite gap, with a gap closing point separating the two phases; the presence or absence of zero energy states in finite length ribbons, where their presence signals a topological state; the topological invariant,
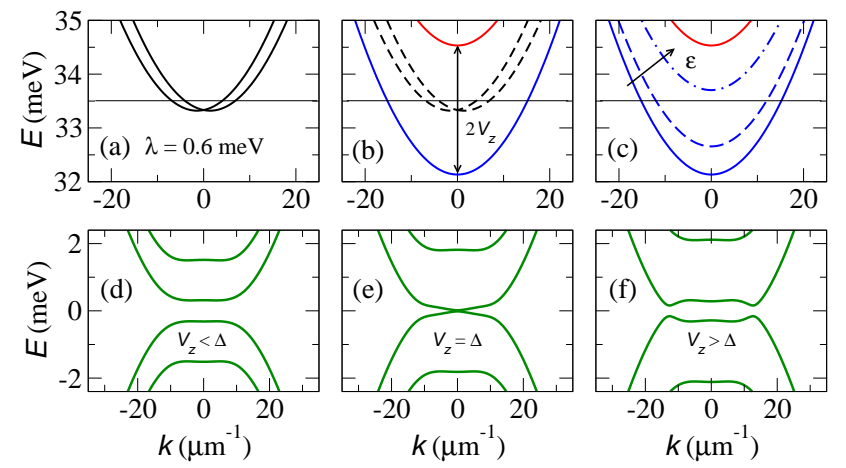

Figure 2: Lowest conduction band for an armchair ribbon of width $N_{y}=81$ unit cells. The effect of Rashba SOC is shown in (a), and the effect of Zeeman coupling in (b), while in (c) we show the evolution of the lowest band with strain, $\varepsilon=0,0.01 \%, 0.03 \%$. For $\Delta \neq 0$ the system develops a gap, which closes at $V_{z}=\Delta(\mathrm{d})-(\mathrm{f})$.

which in this case can be chosen to be the Berry phase $\gamma$ [46], where $\gamma=\pi$ in the topological phase and $\gamma=0$ in the trivial phase [47-49].

Topological superconducting phase.-We consider the lowest conduction band of an insulating armchair ribbon, as in [41], where the valley degeneracy is broken by boundary effects. As shown in [41], and reproduced here for clarity, Rashba SOC lifts spin degeneracy by displacing the parabolic bands horizontally in opposite directions. The psectrum can be seen in Fig. 22(a) for a conservative SOC value $\lambda \simeq 0.6 \mathrm{meV} 30$ 33. The Zeeman coupling lifts the remaining spin degeneracy at $k=0$, opening up a gap of value $2 V_{z}$, as shown in Fig. 2(b). Here we consider the realistic Zeeman field $V_{z} \simeq 1.2 \mathrm{meV}$ (the experimental realization is discussed below). Strain has a strong impact in the system, as shown in Fig. 1(b)1(d), and this is due primarily to strain induced changes in the band structure of the ribbon. This is shown in Fig. 2(c) for strains $\varepsilon=0,0.01 \%, 0.03 \%$. In the present case strain shifts the bands up, but depending on the ribbon width they can also be shifted down [50]. The effect on the topological phase is the same.

In the presence of a finite $s$-wave superconducting pairing $\Delta$ the system becomes gapped, as shown in Fig. 2(d) for $V_{z}<\Delta$. The system goes through a gapless transition point at $V_{z}=\Delta$ [Fig. [2(e)] and becomes gapped again for $V_{z}>\Delta$ [Fig. 2(f)]. In order to determine whether this is a topological transition we have computed the low energy spectrum for a ribbon of finite length, Fig. 3(a)3(c), and the Berry phase $\gamma$, Fig. 3(d)-3(f). As can be seen in Figs. 3(a) and 3(d), or 3(b) and 3(e), the system is topological as long as $V_{z}>\Delta$. For the realistic values $V_{z} \simeq 1.2 \mathrm{meV}$ and $\Delta \simeq 1 \mathrm{meV}$ (experimental realization is discussed below), it is clearly shown in Figs. 33(c) and 3 (f) that a critical strain $\varepsilon_{c}$ exists above which the system becomes topologically trivial. For the parameters used we obtain $\varepsilon_{c} \approx 0.018 \%$ for a ribbon width of 

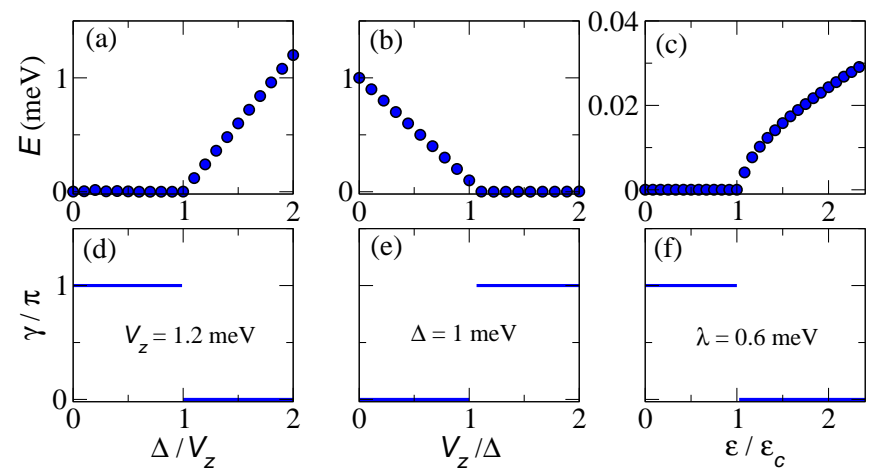

Figure 3: Closest to zero energy mode for a finite ribbon of length $N_{x}=10^{4}$ unit cells $\left(N_{y}=20\right)$ as a function of $\Delta(\mathrm{a})$, $V_{z}(\mathrm{~b})$, and strain $\varepsilon(\mathrm{c})$. Berry phase for the infinite ribbon as a function of $\Delta(\mathrm{d}), V_{z}(\mathrm{e})$, and strain $\varepsilon(\mathrm{f})$.

$\sim 20 \mathrm{~nm}\left(N_{y}=81\right)$, with $\varepsilon_{c}$ not very much dependent on the width $\left(\varepsilon_{c} \approx 0.015 \%\right.$ for a ribbon with $\left.N_{y}=20\right)$. Such a small critical strain $\varepsilon_{c}$ indicates a high sensitivity to lattice deformations, which is primarily due to the effect that strain has on the original band structure. As shown in Fig. 2(b), if we start with the chemical potential $\mu$ inside the $2 V_{z}$ gap, as indicated by the horizontal line, there is then a critical strain for which $\mu$ ceases to be in the gap, as represented in Fig. 2(c). The dependence of the critical strain $\varepsilon_{c}$ with chemical potential $\mu$, Zeeman field $V_{z}$, and strain direction $\theta$, is shown as the phase transition line in the phase diagrams of Figs. 1(b), 1(c), and 1(d), respectively. A high degree of tunability within realistic parameter values is apparent.

Manipulating Majorana zero modes. - The sensitivity to strain found in this work can be used for spatial manipulation of Majorana zero modes. This is demonstrated in Fig. 4, where the armchair ribbon has been subjected to a non-uniform strain profile given by

$$
\varepsilon=\varepsilon_{\max } \frac{1}{2}\left[1+\tanh \left(\frac{x-x_{0}}{\zeta}\right)\right],
$$

where $x$ is the coordinate along the armchair direction, as indicated in Fig. 1(a). Each panel in Fig. 4 shows the Majorana zero mode wave-function amplitude along the ribbon for different profile heights $\varepsilon_{\max }$. If $\varepsilon_{\max }<\varepsilon_{c}$, the two Majorana zero modes appear localized at the two opposite ends of the ribbon (top panel). At $\varepsilon_{\max }=\varepsilon_{c}$ the left side of the ribbon $\left(x>x_{0}\right)$ goes through a topological phase transition, and the gap closes (middle panel). For $\varepsilon_{\max }>\varepsilon_{c}$ the region $x>x_{0}$ becomes trivial, and one of the Majorana zero modes localizes at $x_{0}$ (bottom panel). The Majorana zero mode has thus been transferred to $x_{0}$ through strain manipulation. We used $x_{0}=0.6 a N_{x}$, with $N_{x}=10^{4}$, and $\zeta=0.5 a$, where $a$ is the lattice spacing in the armchair direction.

The advantage of graphene compared to other platforms is that not only strain but also other parameters -

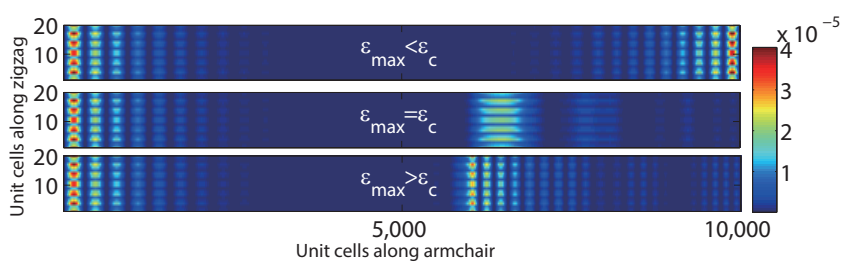

Figure 4: Effect of a strain profile in the Majorana zero mode wave function for three different profile heights parametrized by $\varepsilon_{\max }$ according to Eq. (3).

like the chemical potential $\mu$ and the Zeeman coupling $V_{z}$ - can be manipulated externally, and non-uniform profiles can also be realized for these cases. In particular, by applying a non-uniform chemical potential profile $\tilde{\mu}(x)$ according to

$\tilde{\mu}(x)=\bar{\mu}+\frac{\delta}{2}\left[\tanh \left(\frac{x-\left(x_{0}-l / 2\right)}{\zeta}\right)-\tanh \left(\frac{x-\left(x_{0}+l / 2\right)}{\zeta}\right)\right]$,

the topological region can be confined to a region of length $2 l$ instead of the ribbon's full size. In Fig. 5(a) we show the Majorana zero mode wave-function for different $l$ values. As long as $l$ is large enough to avoid hybridization, it is clear that the two Majorana zero modes localize at the ends of the region of size $2 l$. We used $\bar{\mu}=0.06 \mathrm{meV}$ and $\delta \simeq 0.074 \mathrm{meV}$ in Eq. (4), so that in the central region the chemical potential $\mu=\bar{\mu}+\delta$ is inside the topological gap. A similar effect is observed for a non-uniform Zeeman coupling $\tilde{V}_{z}(x)$. This is shown in Fig. E(b), where a non-uniform profile given by

$\tilde{V}_{z}(x)=\frac{V_{z}}{2}\left[\tanh \left(\frac{x-\left(x_{0}-l / 2\right)}{\zeta}\right)-\tanh \left(\frac{x-\left(x_{0}+l / 2\right)}{\zeta}\right)\right]$,

was used.

Experimental realization.-In order to realize Majorana zero modes in graphene using the standard scheme, and then manipulate them through strain, a sizable superconducting gap and Zeeman field are required, and also needed is a high enough Rashba SOC. We show here that such requirements are within experimental reach.

Regarding the necessary superconducting state, induced superconductivity has been already demonstrated in graphene [51]. An interesting, tempting alternative is the recently discovered superconducting state obtained by decorating graphene with the alkali metal atoms $\mathrm{Ca}$ [52] and Li [53]. Superconductivity has been shown to set in at $T_{c} \sim 6 \mathrm{~K}$, and the measurements suggest a superconducting gap $\Delta \sim 1 \mathrm{meV}$. Moreover, in Ref. [54] it was shown that superconductivity is even not needed if a driven electric field is applied.

Even though superconductivity and moderate to high magnetic fields are usually not compatible, the $2 \mathrm{D}$ nature of graphene assures that only the perpendicular component may weaken the superconducting state. Such perpendicular component $B_{\perp}$ of the magnetic field $B$ can 

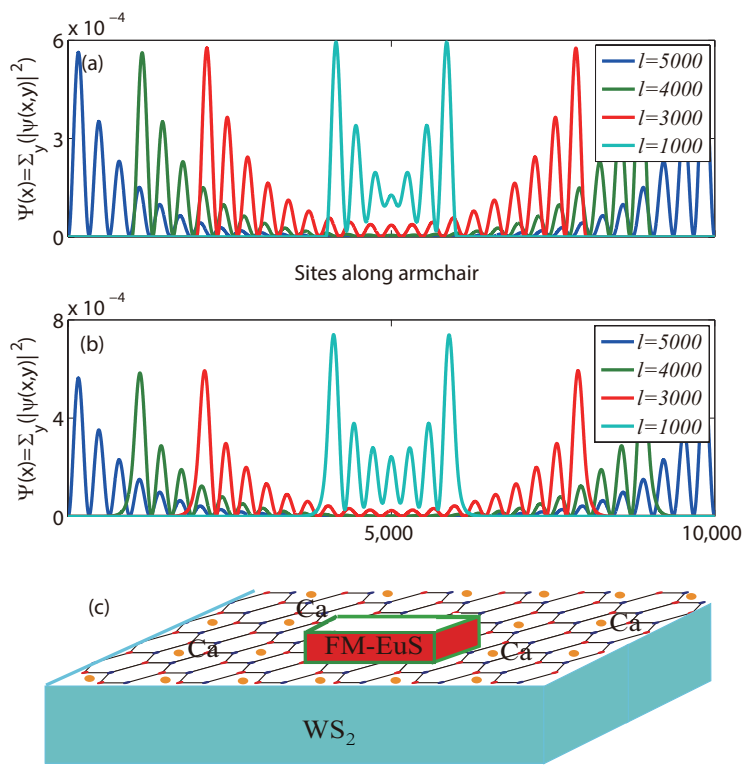

Figure 5: Effect of non-uniform $\mu$ (a) and $V_{z}$ (b) in the Majorana zero mode wave function according to Eqs. (4) and (5), respectively. Parameters: $x_{0}=0.5 a N_{x}$, with $N_{x}=10^{4}$, and $\zeta=0.5 a$, where $a$ is the lattice spacing in the armchair direction. (c) Device proposed in the present work: graphene armchair nanoribbon sandwiched between high SOC transition metal dichalcogenide $\mathrm{WS}_{2}$ and a thin layer of ferromagnetic insulator EuS, decorated with alkali metal atoms $\mathrm{Ca}$ or Li.

be made extremely small in graphene, with $B_{\perp} \lesssim 50 \mathrm{mT}$ for $B=30 \mathrm{~T}$, as shown in recent experiments [55]. Furthermore, we can take advantage of graphene's tunability and use the recently observed Zeeman field $V_{z}$ enhancement by one order of magnitude [36], i.e. $V_{z} \sim 1 \mathrm{meV}$ for $B \gtrsim 1 \mathrm{~T}$, similar to nanowires with enhanced $g$-factor like InAs [11], and fully compatible with the superconducting gap mentioned above. This has been achieved by coupling graphene to the magnetic insulator EuS 36 . The effect has been shown to be operative also on the surface of topological insulators [56] as well as in superconductors [57]. For thin films of EuS the system develops an in-plane easy axis [56], and the effective Zeeman coupling may then be tuned with an in-plane magnetic field, which is the ideal configuration in the present case.

Finally, the Rashba SOC can be enormously enhanced by proximity to $\mathrm{WS}_{2}$ or other transition metal dichalcogenides, reaching values $\lambda \sim 1 \mathrm{meV}[30-32]$ or even higher [33], in agreement with theoretical calculations [58, 59]. Putting all pieces together, we propose the device shown in Fig. 5(c) to realize a topological superconducting state in graphene armchair nanoribbons. Since $\mathrm{WS}_{2}$ and graphene are two-dimensional materials, and given that EuS can be grown in thin films, the effect of small strain may be experimentally investigated using standard tools.
Conclusions. - In the present work we have shown that topological superconductivity can be realized in armchair graphene nanoribbons with externally enhanced parameter values - but still within realistic conditions - using the standard scheme with Rashba SOC, Zeeman field, and $s$-wave superconductivity. A very high sensitivity to strain has been put forward. A non-uniform strain profile can be used to manipulate the position of the localized zero energy Majorana modes. A spatial variation of the chemical potential or Zeeman field can also be used to manipulate Majorana zero modes. Such nonuniform variation of parameters can in principle be easier to achieve in graphene than in other platforms.

It would be interesting to investigate the impact of strain in two recent proposals for realizing Majorana fermions in graphene where SOC enhancement is not required [41, 60]. In Ref. [41], a spatially varying magnetic field was shown to give rise to an additional term in the Hamiltonian which is equivalent to SOC, thus mitigating the lack of SOC in graphene. In Ref. [60], the interaction-induced magnetic ordering of graphene's zero Landau level is shown to give rise to topological superconductivity when the graphene edge is in proximity to a conventional superconductor. No SOC is required in that case. The impact of strain in these two setups may provide an interesting way to manipulate Majorana zero modes.

Z.-H.W. acknowledges support from China Postdoctoral Science Foundation (2016M591057). E.V.C. acknowledges partial support from FCT-Portugal through Grant No. UID/CTM/04540/2013. H.Q.L. and Z.-H.W. acknowledge financial support from NSAF U1530401 and computational resources from the Beijing Computational Science Research Center.

[1] F. Wilczek, Nat. Phys. 5, 614 (2009).

[2] J. Alicea, Rep. Prog. Phys. 75, 076501 (2012).

[3] C. W. J. Beenakker, Annu. Rev. Con. Mat. Phys. 4, 113 (2013).

[4] V. Mourik, K. Zuo, S. Frolov, S. Plissard, E. Bakkers, and L. Kouwenhoven, Science 336, 1003 (2012).

[5] M. Deng, C. Yu, G. Huang, M. Larsson, P. Caroff, and H. Xu, Nano Lett. 12, 6414 (2012).

[6] A. Das, Y. Ronen, Y. Most, Y. Oreg, M. Heiblum, and H. Shtrikman, Nat. Phys. 8, 887 (2012).

[7] L. P. Rokhinson, X. Liu, and J. K. Furdyna, Nat. Phys. 8, 795 (2012).

[8] H. O. H. Churchill, V. Fatemi, K. Grove-Rasmussen, M. T. Deng, P. Caroff, H. Q. Xu, and C. M. Marcus, Phys. Rev. B 87, 241401 (2013).

[9] A. D. K. Finck, D. J. Van Harlingen, P. K. Mohseni, K. Jung, and X. Li, Phys. Rev. Lett. 110, 126406 (2013).

[10] M. T. Deng, C. L. Yu, G. Y. Huang, M. Larsson, P. Caroff, and H. Q. Xu, Sci. Rep. 4, 7261 (2014), 6 pages, 4 figures.

[11] S. M. Albrecht, A. P. Higginbotham, M. Madsen, 
F. Kuemmeth, T. S. Jespersen, J. Nygård, P. Krogstrup, and C. M. Marcus, Nature 531, 206 (2016).

[12] S. Nadj-Perge, I. K. Drozdov, J. Li, H. Chen, S. Jeon, J. Seo, A. H. MacDonald, B. A. Bernevig, and A. Yazdani, Science 346, 602 (2014).

[13] R. Pawlak, M. Kisiel, J. Klinovaja, T. Meier, S. Kawai, T. Glatzel, D. Loss, and E. Meyer, npj Quantum Information 2, 16035 (2016).

[14] Q. L. He, L. Pan, A. L. Stern, E. C. Burks, X. Che, G. Yin, J. Wang, B. Lian, Q. Zhou, E. S. Choi, et al., Science 357, 294 (2017).

[15] D. A. Ivanov, Phys. Rev. Lett. 86, 268 (2001).

[16] M. Leijnse and K. Flensberg, Semicond. Sci. Technol. 27, 124003 (2012).

[17] C. Nayak, S. H. Simon, A. Stern, M. Freedman, and S. Das Sarma, Rev. Mod. Phys. 80, 1083 (2008).

[18] S. D. Sarma, M. Freedman, and C. Nayak, Npj Quantum Information 1, 15001 (2015).

[19] A. H. Castro Neto, F. Guinea, N. M. R. Peres, K. S. Novoselov, and A. K. Geim, Rev. Mod. Phys. 81, 109 (2009).

[20] F. Bonaccorso, L. Colombo, G. Yu, M. Stoller, V. Tozzini, A. C. Ferrari, R. S. Ruoff, and V. Pellegrini, Science 347, 1246501 (2015).

[21] D. Berman, S. A. Deshmukh, S. K. Sankaranarayanan, A. Erdemir, and A. V. Sumant, Science 348, 1118 (2015).

[22] N. Mohanty and V. Berry, Nano letters 8, 4469 (2008).

[23] X.-L. Qi and S.-C. Zhang, Rev. Mod. Phys. 83, 1057 (2011).

[24] J. D. Sau, R. M. Lutchyn, S. Tewari, and S. Das Sarma, Phys. Rev. Lett. 104, 40502 (2010).

[25] J. Alicea, Phys. Rev. B 81, 125318 (2010).

[26] R. M. Lutchyn, J. D. Sau, and S. Das Sarma, Phys. Rev. Lett. 105, 077001 (2010).

[27] Y. Oreg, G. Refael, and F. von Oppen, Phys. Rev. Lett. 105, 177002 (2010).

[28] D. Huertas-Hernando, F. Guinea, and A. Brataas, Phys. Rev. B 74, 155426 (2006).

[29] M. Gmitra, S. Konschuh, C. Ertler, C. Ambrosch-Draxl, and J. Fabian, Phys. Rev. B 80, 235431 (2009).

[30] A. Avsar, J. Y. Tan, J. Balakrishnan, G. K. W. Koon, J. Lahiri, A. Carvalho, A. Rodin, T. Taychatanapat, E. OFarrell, G. Eda, et al., Nature Commun. 5, 4875 (2014).

[31] Z. Wang, D.-K. Ki, H. Chen, H. Berger, A. H. MacDonald, and A. F. Morpurgo, Nature Commun. 6, 8339 (2015).

[32] B. Yang, M.-F. Tu, J. Kim, Y. Wu, H. Wang, J. Alicea, R. Wu, M. Bockrath, and J. Shi, 2D Materials 3, 031012 (2016).

[33] Z. Wang, D.-K. Ki, J. Y. Khoo, D. Mauro, H. Berger, L. S. Levitov, and A. F. Morpurgo, Phys. Rev. X 75, 041020 (2016).

[34] J. Balakrishnan, G. K. W. Koon, M. Jaiswal, A. C. Neto, and B. Özyilmaz, Nature Phys. 9, 284 (2013).

[35] M. Gmitra, D. Kochan, and J. Fabian, Phys. Rev. Lett. 110, 246602 (2013).

[36] P. Wei, S. Lee, F. Lemaitre, L. Pinel, D. Cutaia, W. Cha, F. Katmis, Y. Zhu, D. Heiman, J. Hone, et al., Nature Mater. 15, 711 (2016).
[37] E. V. Castro, H. Ochoa, M. I. Katsnelson, R. V. Gorbachev, D. C. Elias, K. S. Novoselov, A. K. Geim, and F. Guinea, Phys. Rev. Lett. 105, 266601 (2010).

[38] H. Ochoa, E. V. Castro, M. I. Katsnelson, and F. Guinea, Phys. E 44, 963 (2012).

[39] C. H. Lui, L. Liu, K. F. Mak, G. W. Flynn, and T. F. Heinz, Nature 462, 339 (2009).

[40] C. L. Kane and E. J. Mele, Phys. Rev. Lett. 95, 226801 (2005).

[41] J. Klinovaja and D. Loss, Phys. Rev. X 3, 011008 (2013).

[42] M. A. H. Vozmediano, M. I. Katsnelson, and F. Guinea, Phys. Rep. 496, 109 (2010).

[43] B. Amorim, A. Cortijo, F. de Juan, A. G. Grushin, F. Guinea, A. Gutiérrez-Rubio, H. Ochoa, V. Parente, R. Roldán, P. San-José, et al., Phys. Rep. 617, 1 (2016).

[44] V. M. Pereira, A. H. Castro Neto, and N. M. R. Peres, Phys. Rev. B 80, 045401 (2009).

[45] We have used $\beta^{\prime}=-\partial \ln \lambda / \partial \ln a \approx 2$, but this value is largely unknown.

[46] L. Li, E. V. Castro, and P. D. Sacramento, Phys. Rev. B 94, 195419 (2016).

[47] A. P. Schnyder, S. Ryu, A. Furusaki, and A. W. W. Ludwig, Phys. Rev. B 78, 195125 (2008).

[48] S. Ryu, A. Schnyder, A. Furusaki, and A. Ludwig, New J. Phys. 12, 065010 (2010).

[49] Y. Hatsugai, J. Phys. Soc. Jpn. 75, 123601 (2006).

[50] Uniform strain shifts the energy at the M points differently. For tensile strain along armchair, and considering the conduction band, two $M$ points become lower energy while the other increases its energy. In the ribbon, the projected bands can go up or down in energy depending on the type of $\mathrm{M}$ points they are closer to.

[51] H. B. Heersche, P. Jarillo-Herrero, J. B. Oostinga, L. M. K. Vandersypen, and A. F. Morpurgo, Nature 446, 56 (2007).

[52] J. Chapman, Y. Su, C. A. Howard, D. Kundys, A. Grigorenko, F. Guinea, A. K. Geim, I. V. Grigorieva, and R. R. Nair, Sci. Rep. 6, 23254 (2016).

[53] B. Ludbrook, G. Levy, P. Nigge, M. Zonno, M. Schneider, D. Dvorak, C. Veenstra, S. Zhdanovich, D. Wong, P. Dosanjh, et al., PNAS 112, 11795 (2015).

[54] M. Thakurathi, D. Loss, and J. Klinovaja, Phys. Rev. B 95, 155407 (2017).

[55] F. Chiappini, S. Wiedmann, M. Titov, A. K. Geim, R. V. Gorbachev, E. Khestanova, A. Mishchenko, K. S. Novoselov, J. C. Maan, and U. Zeitler, Phys. Rev. B 94, 085302 (2016).

[56] P. Wei, F. Katmis, B. A. Assaf, H. Steinberg, P. JarilloHerrero, D. Heiman, and J. S. Moodera, Phys. Rev. Lett. 110, 186807 (2013).

[57] X. Hao, J. Moodera, and R. Meservey, Phys. Rev. Lett. 67, 1342 (1991).

[58] M. Gmitra and J. Fabian, Phys. Rev. B 92, 155403 (2015).

[59] M. Gmitra, D. Kochan, P. Högl, and J. Fabian, Phys. Rev. B 93, 155104 (2016).

[60] P. San-Jose, J. L. Lado, R. Aguado, F. Guinea, and J. Fernández-Rossier, Phys. Rev. X 5, 041042 (2015). 